\title{
XXVII. Ueber einige im mineralogischen Institute zu München ausgeführte Untersuchungen.
}

\author{
Von \\ C. Viola in Rom. \\ (Hierzu Tafel VII und VIII und 7 Textfiguren.)
}

Inhalt. I. Das Czapski's che Goniometer. Prüfung und Berichtigung. S. 418. Die Autocollimation. S. 421. Das Arbeiten mit dem Theodolithgoniometer. S. 422. Albit von Lakous. S. 423. - II. Das Abbe'sche Refractometer. S. 427. Prüfung und Berichtigung. S. 428. Der Nullpunkt des Verticalkreises. S. 431. Das Arbeiten mit dern $\mathrm{A}$ b be'schen Refractometer. S. 482. Brechungsvermb̈gen und Dispersion des A b be'schen Glases. S. 435. Brechungsindices und Dispersion des Albits von Lakous. S. 436. Genauig-• keit der Resultate. S. 437. - III. Di e optische Bestimmung nach Fedorow. S.439.

Bei meinem diesjahrigen Aufenthalte in Munchen hatte ich Gelegenheit, folgende neue Apparate zu benutzen:

1. ein Theodolithgoniometer nach Gapski, construirt von R. Fuess;

2. das von Dr. Pulfrich $\left.{ }^{1}\right)$ neuconstruirte $A b b e^{\prime}$ sche Refractometer von der bekanten Firma Carl Zeiss in Jena, und

3. das grosse Mikroskop mit drehbaren Nicols und Fedorow'schem Tische von R. Fuess in Berlin.

Indem ich uber die Untersuchungen, welche ich mit den soeben genannten Apparaten gemacht habe, berichten möchte, füle ich mich gedrungen, Herrn Prof. Groth meinen besten Dank auszusprechen.

Ich habe schon vor ein paar Jahren etliche schöne Krystalle von Albit von Lakous (Insel Kreta) untersucht und studirt ${ }^{2}$ ), habe aber die optischen Untersuchungen derselben absichtlich auf spätere Zeit verschoben, da die damals mir zur Verfugung stehenden Mittel nicht geeignet schienen, um mit Nutzen und Erfolg die Untersuchungen vorzunehmen. Auch verfugt leider das mineralogische Institut in Rom nicht uber solche Instrumente,

1) Eine ausführliche Beschreibung dieser Nंeuconstruction von Hrn. Dr. Pulfrich wird im nåchsten Hefte dieser Zeitschrift erscheinen.

2) C. Viola, Ueber den Albit von Lakous (Insel Kreta) mit chemischer Analyse von E. Mattirolo. Tschermak's Mineral. und petrogr. Mittheil. 15, 135. Ref. diese Zeitschr. 29, 151. 
welche grosse Genauigkeit beanspruchen könnten. Ich habe daher keine bessere Gelegenheit finden können, als eben von der Güte von Prof. Groth Gebrauch zu machen.

Ich werde also in der Folge uber den Albit von Lakous berichten; dabei setze ich natürlich das, was ich uber denselben Feldspath schon mitgetheilt habe, voraus.

Von allen jenen Albitkrystallen, welche ich s. Z. für meine Studien an der Hand hatte und deren grösster Theil, jetzt im mineralogischen Institute von Bologna aufbewahrt, abgebildet wurde, sind mir zwei schöne Kryställchen zurückgeblieben, die mit denen in Fig. 1 und Fig. 2 abgebildeten ${ }^{1)}$ grosse Aehnlichkeit haben. Ihre Flächen sind sehr gestreift und gekrummt, und eignen sich daher fur die Messung mit dem Theodolithgoniometer.

\section{Das Czapski'sche Goniometer.}

Das Theodolithgoniometer, wie es jetzt von Fuess construirt wird, bestebt aus zwei aufeinander senkrechten Drehaxen, welche unbeweglich sind; die eine Axe ist vertical und trägt die Justirvorrichtung zur Aufstellung des Krystalles, die andere ist horizontal und trägt das Fernrohr und das mit ihm verbundene Signal. Die Drebungswinkel werden auf einem horizontalen und einem verticalen Kreise abgelesen.

Der Vortheil dieser Einrichtung besteht darin, dass beide Drehaxen fest sind, und dennoch die Möglichkeit vorhanden ist, die Reflexe des Signales durch jede Krystallfläche zu erhalten.

Das Signal besteht aus einem in Stanniolpapier eingeschnittenen Kreuz, welches in der Ebene des Fadenkreuzes des Oculars placirt ist und mit Húlfe eines elektrischen Glublampchens beleuchtet wird.

Nachdem eine solche Einrichtung getroffen ist, ist es klar, dass, wenn das Fernrohr für im Unendlichen befindliche Objecte eingerichtet ist, das Signal selbst ins Unendliche geruckt wird.

So oft das von einer Krystallfäche in Unendlich befindliche Signal reflectirt wird und genau im Kreuzpunkte des Oculares einspielt, tritt der Pol der Krystallfläche immer in einen bestimmten Meridian ein, der zum Verticalkreise parallel ist und zum Horizontalkreise senkrecht steht. Auf dieses Princip stutzt sich die Messung und die Genauigkeit des Goniometers.

\section{Prüfung und Berichtigung.}

Denken wir uns, dass der Reflex einer Krystallfäche genau in den Kreuzpunkt des Oculars einspiele.

Dreht man nun den Krystall um seine verlicale Axe um genau $180^{\circ}$ und schlägt man das Fernrohr um, so wird auch jetzt, durch richtige Stel-

1) 1. c. Taf. IV. 
Iung des Fernrohres, der Reflex des Signales wieder in den Kreuzpunkt einspielen müssen, falls das Instrument genau berichtigt ist.

Betrachten wir den vom Signal einfallenden und den von der Krystallfläche reflectirten Lichtstrahl, welche mit der Visirlinie zusammenfallen. Die Mittellinie dieser zwei Strahlen ist offenbar gleichzeitig die Normale der Krystallfäche, welche wir auch Collimationsnormale nennen können; sie muss zur Drehaxe des Fernrobres senkrecht stehen, wenn der aufgestellten Forderung genúgt werden soll.

Wenn die Collimationsnormale zur Drehaxe des Fernrohres senkrecht und wenn wiederum diese zur Drehaxe des Krystalles senkrecht steht, so werden, durch Einfuhrung der Reflexe auf den Kreuzpunkt des Oculars, sämmtliche Flächenpole des Krystalles nach und nach in einen und denselben bekannten Meridian gebracht; so wird durch die Ablesung auf dem Verticalkreise die Lage der Flächen im Meridian und durch die Ablesung auf dem Horizontalkreise die Lage der zu den Flächen gehörenden Meridiane festgestellt.

Wir sehen somit, dass wir folgende zwei Punkte des Goniometers zu prufen und zu berichtigen haben:

1. die Collimationsnormale muss zur Horizontaldrehaxe senkrecht stehen;

2. die Horizontaldrehaxe muss normal auf der Verticaldrehaxe sein.

Ad 1. Die Collimationsnormale mache einen Fehlerwinkel $\varepsilon_{1}$ mit der Normalen zur Drehaxe des Fernrohres.

Wir gelangen zur Kenntniss dieses Fehlerwinkels durch folgende Prufung. Man lege das Fernrohr horizontal, d. b. senkrecht zur Verticalaxe des Goniomelers, und suche das durch eine Verticalfläche reflectirte Signal. Man kann z. B. anstatt einer Krystallfläche bequemer und einfacher ein planparalleles Glas anwenden. Eine solche Fläche steht zur Verticalaxe des Goniometers parallel, wenn durch Drehen derselben um $180^{\circ}$ der Reflex des Signals wieder in's Fadenkreuz des Oculars eintritt. Ist das einmal gepruft, so wird das Fernrohr umgeschlagen und noch einmal horizontal gestellt. Ist der Fehler $\varepsilon_{1}$ vorhanden, so wird das reflectirte Signal nicht mehr in den Kreuzpunkt des Oculars einspielen; es wird vielmehr eine kleine Differenz zum Vorschein kommen, welche genau $2 \varepsilon_{1}$ beträgt. Die Hälfte dieser Differenz, d. h. der Fehlerwinkel $\varepsilon_{1}$, wird durch Verschiebung des Fadenkreuzes oder des am Objectiv angebrachten Prismas eliminirt. Die Prufung und Berichtigung muss naturlich wiederholt werden, bis kein Fehler $\varepsilon_{1}$ zu constatiren ist.

Da das Objectiv sammt Prisma des Fuess'schen Apparates mehrmals abgenommen werden musste, um eine Papierblende im Tubus einzuschieben, so wurde eine Prüfung und Berichtigung des Goniometers nothwendig, was durch die hier angedeutete Melhode vorgenommen werden konnte. 
Ad 2. Wenn die Drehaxe des Fernrohres nicht genau senkrecht zur Drehaxe des Krystalles steht, so liegt dieser Fehler in der Gonstruction des Goniometers und kann nur durch den Mechaniker weggebracht we den. Ich bezeichne ibn mit $\varepsilon$, Fig. 1 .

Durch das Auftreten von $\varepsilon$ bewegt sich die Gollimationsnormale nicht in einer durch die Verticalaxe gehenden Meridianebene, sondern in einer

Fig. 1.

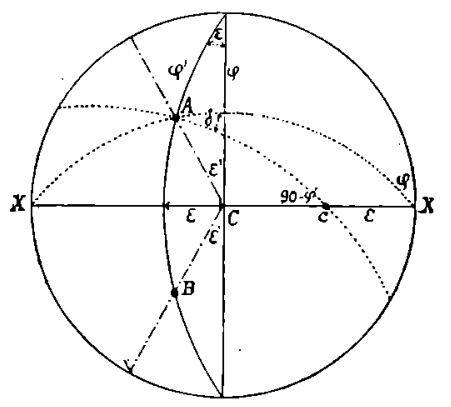
solchen, welche mit der verticalen Drehaxe einen Winkel $\varepsilon$ einschliesst. Dadurch werden sowohl die am Verticalkreise als auch die am Horizontalkreise abgelesenen Winkel falsch. Vorerst ist leicht zu sehen, dass der Fehlerwinkel $\varepsilon$ keinen Einfluss bei der Prufung des Fehlers $\varepsilon_{1}-$ ad 1 - ausubt, wenn das Fernrobr horizontal gebalten wird. Um $\varepsilon$ zu bestimmen, mussen wir vorher $\varepsilon_{1}$ wegschaffen.

Der Fehlerwinkel $\varepsilon$ würde sofort bekannt, wenn wir das Fernrohr nach oben vertical richten wurden, das in einer Horizontalläche gespiegelte Signal in's Fadenkreuz einstellten und die kleine Abweichung im Oculare sofort ablesen könnten. Da aber das vorliegende Goniometer nicht dafür eingerichtet ist, so verfahren wir auf folgende Weise.

Wir stellen das Fernrohr etwas schief und richten eine Fläche so ein, dass der Reflex des Signals im Kreuzpunkte des Oculars genau einspielt.

Es sei $A$, Fig. 1 , der Pol der angenommenen Fläche und $\varphi^{\prime}$ der abgelesene Höhenwinkel.

Das Fernrohr wird umgeschlagen und wieder auf den Höhenwinkel $\varphi^{\prime}$ eingestellt. Durch Drehen der spiegelnden Fläche um ungefähr $180^{\circ}$ wird das Signal wieder in Coincidenz mit dem Kreuzpunkte gebracht und der Horizontalwinkel abgelesen. Die Differenz von $180^{\circ}$ ist der doppelte Fehlerwinkel $2 \varepsilon^{\prime}$. Aus der Gleichung

$$
\sin \varepsilon=\frac{\operatorname{tg} \varepsilon^{\prime}}{\operatorname{tg} \varphi^{\prime}}
$$

wird $\varepsilon$ berechnet. Daraus ergiebt sich aber auch

$$
\operatorname{tg} \varphi=-\cos \varepsilon \cdot \operatorname{tg} \varphi^{\prime} .
$$

Der wahre Höhenwinkel $\varphi$ făllt etwas grösser aus, als der abgelesene Winkel $\varphi^{\prime}$.

Bei mir war $\varphi^{\prime}=45^{\circ} 20^{\prime}$, der abgelesene Fehler aber nur $2 \varepsilon^{\prime}=4^{\prime}$, also $\varepsilon^{\prime}=2^{\prime}$; das entspricht circa $\varepsilon=2^{\prime}$. Erst für einen Höhenwinkel von $\varphi^{\prime}=88^{\circ}$ wird man einen Fehler von circa $1^{0}$ der auf dem Horizontalkreise abgelesenen Winkel constatiren können. Dagegen wird der Fehler 
auf dem Verticalkreise in Folge von $\varepsilon=2^{\prime}$ immer kleiner sein als $\mathbf{2}^{\prime}$, was offenbar vernachlässigt werden muss.

Diese Genauigkeit in der Construction des Capski'schen Goniometers gereicht der Firma R. Fuess und dem Leiter der betreffenden Abtheilung, Herrn Leiss in Berlin, zur Ehre.

\section{Die Autocollimation.}

Allerdings haben sich im Laufe der Arbeit doch bei dem hier in Rede stehenden Goniometer verschiedene Unannehmlichkeiten herausgestellt, welche seine Brauchbarkeit für die meisten Messungen geradezu unmöglich machen, und ich freue mich daruber, dass auch Herr Prof. Groth bei der Prufung des Instrumentes dieselbe Meinung äusserte.

Eine erste Unannehmlichkeit ist die Autocollimation, wie sie bei dem Fuess'schen Goniometer zum Ausdruck gebracht wurde.

Um Messungen vorzunehmen, mussen wir vorerst die spiegelnde Fläche grob in die verlangte Lage einstellen können. Es hat sich aber herausgestellt, dass durch Einschalten der Lupe das Signal nicht vom Unendlichen her sein Licht sendet, sondern von einer Stelle, welche mit der Krystallfläche genau zusammenfällt. Und da das Signal schmal ist, so wird nur ein Theil der Fläche beleuchtet, oder auch gar nichts, wenn diese vom Centrum der Drehaxe um etwas veriuckt ist. Die Folge davon ist, dass das Aufsuchen einer Fläche ungemein schwer wird.

Nachtheilig wirkt aber hierbei ausserdem das stark beleuchtete Gesichtsfeld des Fernrobres, was dadurch entsteht, dass das am Objectiv angebrachte Prisma viel Licht des Signals zuruckstrahlt.

Man würde solche Unannehmlichkeiten vermeiden können, ohne die Autocollimation wegzuschaffen, wenn das Signal ausserhalb des Objectives gebracht und die Lupe nicht vor dem Signale, sondern nur vor der Objectivlinse eingeschaltet werden könnte.

Allein es frägt sich: ist die Autocollimation nothwendig oder durchaus erforderlich fur die Messung eines Krystalles?

Wir wissen, dass die Autocollimation nur da, besonders in der Geodäsie und Astronomie, ausgezeichnete Dienste leistet, wo keine andere Beleuchtung oder gar keine andere Beobachtungsart möglich isı, oder wo das Fadenkreuz selbst (Talcott'sche Methode) die Rolle des Objectes spielen muss.

In der Krystallographie aber, und zwar bei der Feststellung der Lage einer Fläche, ist fưr die Deutlichkeit der Reflexe eine schiefe Beleuchtung unbedingt vorzuziehen. Sie kann aber auch in dem $\mathrm{Czapski}$ 'schen Goniometer mit Leichtigkeit eingefuhrt werden 1 ).

1) Herr Leiss berichtete mir inzwischen, dass eine derartig geänderte Construction des Instrumentes bereits ausgeführt sel. 
Durch die schiefe Beleuchtung kommen die einzelnen Eigenschaften einer Fläche zum Vorschein, werden die anstossenden Flächen auch theilweise gesehen, was das Aufsuchen einer Fläche entschieden erleichtert.

\section{Das Arbeiten mit dem Theodolithgoniometer.}

Ich habe verschiedene Messungen sowohl an Quarzkrystallen, als auch an Albitkrystâllchen mit dem $\mathrm{Cz}$ apsk i' schen Goniometer vorgenommen.

Die zur trigonalen Axe parallelen Quarzflächen sind streng genommen aus einer Anzahl kleiner Flächen zusammengesetzt, welche die bekannte und charakteristische Streifung ausmachen. Die diese Streifung bildenden Flächen schliessen einen Winkel ein, der höchstens $6^{0}$ und meistens nur einige Minuten beträgt.

Die zu dieser Streifung gehörende Zone ist nicht immer dieselbe und senkrecht zur trigonalen Axe, sondern schief etwa um $2^{\circ}$ herum. Auch die Pyramidenflächen haben verschiedene Reflexe gegeben.

Mit dem Theodolithgoniometer sind solche Streifungen leicht zu bestimmen, auch krumme Flächen lassen sich damit bequem studiren. Alle hierher gebörenden Reflexe kommen fast gleichzeitig in's Gesichtsfeld; durch die zwei Drehbewegungen ist es eine Kleinigkeit, sie nach und nach auf den Kreuzpunkt zu bringen. Auch kann man gewöhnlich muhelos die richtigen Reflexe von den sogenannten falschen unterscheiden, nämlich solche, die durch Beugung des Lichtes entstehen, da diese gefärbt sind, wenn weisses Licht zur Anwendung kommt.

Um die hier geschilderten Arbeiten vorzunehmen, brauchen wir eigentlich kein Theodolithgoniometer. Ein gewöhnliches Babinet'sches Goniometer genúgt hierzu vollkommen; man denke nur, dass das Ocular mit einem Mikrometer versehen ist, das Minuten und Secunden anzugeben vermag. Ich glaube eine solche Einrichtung ist schon von Groth vorgeschlagen worden, um die von einer Zone abweichenden Flächen zu bestimmen; sie vervollständigt das mit einer Drehaxe versehene Goniometer und ersetzt dadurch unbedingt ein Theodolithgoniometer ${ }^{1}$ ).

Wir haben gesagt, dass es leicht und bequem ist, die Lage aller solcher Reflexe festzustellen, welche in's Gesichtsfeld des Oculars fallen. Handelt es sich aber darum, mit dem Theodolithgoniometer nach Reflexen zu spuren, welche weit auseinander liegen und von sehr kleinen und schlechten Flăchen gebildet werden, so ist dies eigentlich eine mubsame und undankbare, wenn man nicht sagen will, eine vergebliche Arbeit. Treten wir näher in die Frage ein.

1) Ein solches Mikrometer hat inzwischen Herr Leiss für das gewöhnlich gebrauchte Ocular eines Goniometers II $\mathrm{Fu}$ es $s$ des min. Instiluts in München ausgeführt; ein 'Theilstrich desselben entspricht $10^{\prime}$ (mit einem Fehler von nur $\pm 5^{\prime \prime}$ ), und die Theilung gebt nach oben und unten bis auf einen Abstand von $3^{0}$. 
Bei dem Theodolithgoniometer handelt es sich stets um zwei Drehaxen, die gleichzeitig bewegt werden mussen, sei es, dass beide Axen zum Krystall, oder dass eine zum Krystall und die andere zum Fernrohr gehöre. Da keine grobe Einstellung vorbanden ist, so kann mit Leichtigkeit eine kleine Fläche ubergangen werden. Wir bemerken, dass beim Theodolithgoniometer ein Umstand auftritt, der darin besteht, Kanten und Zonen, also das, was einen Krystall besonders auszeichnet, zu vernachlässigen.

Will man z. B. eine Ebene in eine bestimmte Lage bringen, und verftgt man uber zwei aufeinander senkrechte Drehbewegungen, so ist es naturlich, dass man, wenn rasch gearbeitet werden soll, eine in der Ebene liegende Gerade zuerst in parallele Lage zu einer der beiden Drehaxen bringt, damit eine sichere Drehbewegung bewirkt werden kann. Bei den Krystallen sind solche gerade Linien offenbar zablreich vorhanden; sie stellen die Zonen dar, um welche herum die Flächen entwickelt sind. Das Theodolithgoniometer kann sie gar nicht benutzen; auch wtirde sonst die eigentliche Theodolitharbeit verloren gehen, welche darin besteht, nur Pole in Bezug auf zwei Drehaxen festzustellen.

Ich bin der Meinung, dass, sowohl was die Messung der Krystalle, als als auch was die Signatur der Flächen anbetrifft, man hauptsächlich auf die Zonen Rucksicht haben muss, denn nur dadurch kann eine Erleichterung herbeigefuhrt werden, obwohl die meisten Flächen von den Zonen ein wenig abweichen.

Das Princip der Zonen, oder was gleich ist, das Princip der Rationalität darf nicht bei Seite gelassen werden, wiewohl es in der Natur nicht mit absoluter Strenge zutrifft. Ein solches Princip ist eben der Leitfaden der Krystalle, wie das New to n'sche Gesetz, das auch nicht genau in der Natur gilt, fur die Bahnberechnung der Planeten jedoch das Fundament bildet.

Erfullt ein Theodolitbgoniometer solche Bedingungen nicht, so hat es besseren Instrumenten Platz zu machen.

Albit von Lakous. Ich habe schon s. Z. die chemische Zusammensetzung des Albits von Lakous angegeben, wie sie aus der Analyse von Mattirolo zu schliessen ist ${ }^{1}$ ):

\begin{tabular}{lr}
$\mathrm{SiO}_{2}$ & 68,51 \\
$\mathrm{Al}_{2} \mathrm{O}_{3}$ & 19,83 \\
$\mathrm{Na}_{2} \mathrm{O}$ & 11,74 \\
$\mathrm{~K}_{2} \mathrm{O}$ & 0,16 \\
Gluhverlust & 0.15 \\
\cline { 2 - 2 } & 100,39
\end{tabular}

und dieser stellt neben demjenigen vom Kasbek, wie schon damals bemerkt worden ist, den reinsten bekannten Albit dar.

1) I. c. S. 158 . 
Wegen dieses Umstandes babe ich die Studien uber den Albit von Lakous wieder aufgenommen.

Ich verfugte uber zwei wasserhelle, ganze Albitkryställchen, welche denen der schon publicirten Fig. 1 und 2 sebr ähnlich sind.

Beide Kryställchen bestehen aus zwei Zwillingen nach dem Albitgesetze; im Dunnschliffe treten aber mebrere Zwillingsstreifen auf, welche die Schwierigkeiten fur ein genaues optisches Studium des Feldspathes bedeutend vermehren.

Die vorherrschenden Flächen sind: $(0 \bar{T} 0),(1 \overline{3} 0),(1 \bar{T} 0),(021),(110)$, $(130),(001)$ und $(00 \bar{T})$ eines Individuums, und (이 0$) ;(\underline{T} 30),(\underline{T 10}), \underline{00 T})$

Fig. 2.

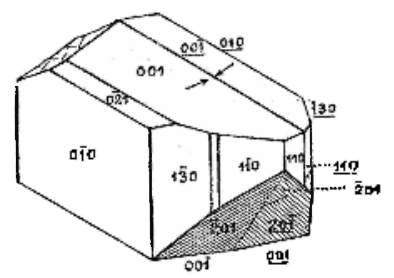
und (001) des anderen. Die nebensteh ende Figur (Fig. 2) giebt angenäbert einen dieser Albitzwillinge wieder. Die gestreiften und matten Flächen (201) und (20T) baben keine Reflexe mit dem gewöhnlichen und noch weniger mit dem Theodolithgoniometer gegeben.

Die vorhin bezeichneten Flächen sind aber streng genommen nicht als solche aufzufassen. Sie bestehen vielmehr aus einer grossen Anzahl von kleinen Flächen, welche sowohl die Krummung als auch die Streifung der genannten Flächen bilden. Als deutliche Flächen können nur (110), (021), (001) und (00T) angesehen werden.

Es wird wohl interessant sein, dass ich die Lage der Reflexe, die ich mit dem Czapski'schen Goniometer und mit dem gewöhnlichen Goniometer controlirt habe, angebe.

Um die abgelesenen Winkel sofort auf ein stereographisches Netz aufzutragen, wie ein Feldspath gewöbnlich orientirt wird, babe ich die Zone [001] oder diejenige, welche als solche angegeben werden konnte, parallel zur verticalen Drehaxe gestellt. Das war naturlich nicht leicht zu erreichen, da die Flächen $(0 \bar{T} 0),(1 \overline{3} 0),(1 \bar{T} 0),(110),(130),(\underline{T 30})$ und $(\underline{010})$ keine einbeitliche Zone bilden. Doch konnte man durch sorgfältige Justirung des Krystalles erreichen, dass die deutlichsten Reflexe von (0T0), (1T0) und (110) im Fadenkreuz spielten während der Drehung der Verticalaxe und horizontalen Stellung des Fernrobres. Diese horizontale Lage wurde als Nullpunkt des Verticalkreises angenommen. Der Nullpunkt des Horizontalkreises konnte naturlich beliebig sein. Um aber die Sache zu vereinfachen und die Rechnung zu vermeiden, wurde der Nullpunkt mit dem deutlichsten Reflexe der Fläche (0ग0) in Uebereinstimmung gebracht.

Nach dieser Auseinandersetzung wird die folgende Zusammenstellung verständlich erscheinen : 
Ueber einige im mineral. Institute zu München ausgeführte Untersuchungen. 425

$$
\text { Reflexe der Albitflächen. }
$$

\begin{tabular}{|c|c|c|c|}
\hline $\begin{array}{r}\text { Horizon } \\
352\end{array}$ & $\begin{array}{l}\text { talkreis : } \\
050^{\prime}\end{array}$ & $\begin{array}{l}\text { Verticalkreis: } \\
\quad 0^{0} 36,5^{\prime}\end{array}$ & $\begin{array}{c}\text { Gewicht: } \\
1\end{array}$ \\
\hline 353 & 22 & 029,5 & $1 \frac{1}{2}$ \\
\hline 353 & 45 & $\begin{array}{l}029,5 \\
5\end{array}$ & $1 \frac{1}{2}$ \\
\hline 354 & 49 & $0 \quad 29,5$ & $1 \frac{1}{2}$ \\
\hline 355 & 0 & 020,5 & 2 \\
\hline 356 & 30 & $0 \quad 20,5$ & 2 \\
\hline 356 & 53 & 020,5 & 2 \\
\hline 357 & 17 & 020,5 & 2 \\
\hline 0 & 0 & 00 & 8 \\
\hline 0 & 28 & $0 \quad 12$ & 4 \\
\hline 0 & 46 & 012 & 4 \\
\hline & 1,5 & $\begin{array}{ll}0 & 12\end{array}$ & 4 \\
\hline 1 & 17 & $\begin{array}{ll}0 & 12\end{array}$ & 4 \\
\hline 1 & 38,5 & 020,5 & 2 \\
\hline 8 & 38 & $0 \quad 36,5$ & 1 \\
\hline 25 & 1 & $\begin{array}{ll}0 & 10\end{array}$ & 1 \\
\hline 25 & 19 & 08 & 4 \\
\hline 26 & 1 & $0 \quad 6$ & 2 \\
\hline 26 & 54,5 & $\begin{array}{ll}0 & 0\end{array}$ & 1 \\
\hline 29 & 57 & $\begin{array}{ll}0 & 0\end{array}$ & 1 \\
\hline 30 & 8 & $\begin{array}{ll}0 & 0\end{array}$ & 1 \\
\hline 30 & 27,5 & $\begin{array}{ll}0 & 5\end{array}$ & 8 \\
\hline 30 & 18 & 05 & 8 \\
\hline 30 & 33 & 04 & 1 \\
\hline 30 & 45,5 & $\begin{array}{ll}0 & 6\end{array}$ & 8 \\
\hline 30 & 57 & $\begin{array}{ll}0 & 7\end{array}$ & 2 \\
\hline 33 & 48 & $\begin{array}{ll}0 & 10\end{array}$ & $1 \frac{1}{2}$ \\
\hline 60 & 0 & $\begin{array}{ll}0 & 8\end{array}$ & 3 \\
\hline 60 & 4,5 & $\begin{array}{ll}0 & 0\end{array}$ & 8 \\
\hline 60 & 21,5 & $\begin{array}{ll}0 & 8\end{array}$ & 3 \\
\hline 116 & 39,5 & $\begin{array}{ll}0 & 12\end{array}$ & 1 \\
\hline 117 & 47 & $\begin{array}{ll}0 & 10\end{array}$ & $\frac{1}{2}$ \\
\hline 118 & 33 & $\begin{array}{ll}0 & 0\end{array}$ & 9 \\
\hline 119 & 34 & $\begin{array}{ll}0 & 0\end{array}$ & $\frac{1}{2}$ \\
\hline 120 & 0 & 35950 & 3 \\
\hline 120 & 16 & $359 \quad 45$ & 3 \\
\hline 120 & 39,5 & 35950 & 3 \\
\hline
\end{tabular}




\begin{tabular}{|c|c|c|c|}
\hline \multicolumn{2}{|c|}{ Horizontalkreis. } & Verticalkreis: & \multirow{2}{*}{$\begin{array}{c}\text { Gewicht: } \\
\mathbf{3}\end{array}$} \\
\hline 1210 & $8^{\prime}$ & $359^{\circ} 52$ & \\
\hline $121:$ & 39 & 35952 & 1 \\
\hline 149 & 13 & 00 & $1 \frac{1}{2}$ \\
\hline 149 & 29 & 35955 & 8 \\
\hline 149 & 38 & 35955 & 4 \\
\hline 149 & 50 & 35950 & 4 \\
\hline 150 & 1 & 35950 & 4 \\
\hline 153 & 2 & $\begin{array}{ll}0 & 0\end{array}$ & 1 \\
\hline 153 & 38 & 04 & 1 \\
\hline 154 & 18 & 06 & 1 \\
\hline 154 & 48 & 06 & 1 \\
\hline 155 & 21 & $\begin{array}{ll}0 & 8\end{array}$ & $1 \frac{1}{2}$ \\
\hline 179 & 33 & 010 & $1 \frac{1}{2}$ \\
\hline 181 & 21 & 020 & 1 \\
\hline 183 & 20 & 040 & 1 \\
\hline 184 & 42 & 150 & 1 \\
\hline 22 & 33 & 4110 & 8 \\
\hline $\mathbf{2 3}$ & 10 & 4045 & 6 \\
\hline 24 & 0 & 4020 & 6 \\
\hline 25 & 0 & $40 \quad 0$ & $\mathbf{5}$ \\
\hline 80 & 15 & 6335 & 6 \\
\hline 97 & 35 & 650 & $\mathbf{5}$ \\
\hline 97 & 50 & 6435 & $\mathbf{5}$ \\
\hline 98 & 10 & 640 & 7 \\
\hline 98 & 33 & 6320 & 7 \\
\hline 99 & 0 & 620 & 7 \\
\hline
\end{tabular}

Aus dieser Zusammenstellung gebt deutlich hervor, dass zur Individualisirung beider Zonen [001] und [100] die vorhandenen Ablesungen vollständig genügen. Als Mittel findet man :

$$
\beta=116^{\circ} 20^{\prime} \text {. }
$$

Zur besseren Orientirung der einzelnen Pole von Albit habe ich das stereographische Diagramm beigegeben, Taf. VII.

Man sieht, dass fur die Bestimmung der zwei anderen Fundamentalwinkel $\alpha$ und $\gamma$ die Flächen (0T0), (1T0), (110) und (130) am geeignetsten erscheinen, wenn man darunter die deutlichsten Reflexe versteht.

Machen wir zwei Annabmen. 
Ueber einige im mineral. Instilute zu München ausgeführte Untersuchungen. 427

1) Die Flächen (0T0), (1T0) und (110) werden zu Grunde gelegt; so erhält man :

$$
(0 T 0):(100)=89^{\circ} 7,6^{\prime} \text {. }
$$

2) Die drei Flächen (0T0), (1T0) und (130) werden fur die Berechnung ausgewählt; so ergiebt sich:

$$
(0 \mathrm{~T} 0):(100)=89^{\circ} 48,2^{\prime} \text {. }
$$

Aus den Reflexen, welche die Flachen (001) und (미) gegeben haben, geht als Mittel der Winkel

hervor.

$$
(0 \pi 0):(001)=93^{\circ} 41^{\prime}
$$

Für die erste Annabme findet man:

fur die zweite:

$$
\begin{aligned}
& \alpha=94^{\circ} 33^{\prime} \\
& \gamma=879
\end{aligned}
$$

$$
\begin{aligned}
& \alpha=94^{\circ} 25^{\prime} \\
& \gamma=8821
\end{aligned}
$$

Als Mittelwerthe durfen wir annehmen:

$$
\begin{aligned}
(0 \mathrm{~T} 0):(100) & =89^{\circ} 27,9^{\prime} \\
\alpha & =9423,2 \\
\gamma & =8733,4
\end{aligned}
$$

Aus einer grossen Anzahl von Albitkrystallen habe ich seiner Zeit Folgendes gefunden:

und

$$
\begin{aligned}
& \alpha=94014,50^{\prime} \\
& \beta=116 \quad 31,75 \\
& \gamma=88 \quad 5,16
\end{aligned}
$$

bestimmt.

$$
a: b: c=0,635: 1: 0,557
$$

\section{Das Abbe'sche Refractometer.}

Um die Lichtbrechungsindices des Albits zu bestimmen, gebrauchte ich vorerst das Liebisch'sche Refractometer, ferner das von R. Fuess construirte und dem Czapski'schen Theodolithgoniometer angepasste Abbesche Refractometer. Ich theile aber die dadurch erhaltenen Resultate nicht mit, da ihre Genauigkeit keinen Vergleich aushält mit derjenigen der mit dem von C. Zeiss in Jena construirten Abbe-Pulfrich'schen Refractometer bestimmten Resultate.

An diesem ausgezeichneten Apparate sind alle Theile, sowohl die rein mechanischen als auch die optischen, so berechnet und bemessen, dass das Arbeiten damit ein ausserordentlich bequemes, rasches und genaues genannt werden kann. 
Das Objectiv ist coneav und an die Halbkugel fast genau angepasst, so dass das von der Halbkugel herkommende Licht obne Verlust und ohne Verunreinigung durch das Fernrohr aufgenommen werden kann. Das Licht wird nur auf das zu bestimmende Mineral concentrirt, so dass das Feld des Fernrohres nicht ubertrieben beleuchtet erscheint. Diese günstigen Umstände und die Reinheit der Linsen wirken dahin, dass die Grenzlinien mit einer ungewöbnlichen Schärfe zum Vorschein kommen, und zwar so, dass ohne Anwendung des Nicols gleichzeitig beobachtet und eingestellt werden kann, auch dann, wenn die verwendete Fläche des Minerals nur 1 qmm beträgt.

\section{Prüfung und Berichtigung.}

1. Die obere Ebenenfläche der Halbkugel muss zu ihrer Rotationsaxe senkrecht stehen;

2. diese letztere normal zur Drehaxe des Fernrohres;

3. die Visirlinie des Fernrohres muss zu ihrer Drehaxe senkrecht stehen.

Ad 1. Wir halten das etwas geneigte Fernrohr fest, und wenn es nöthig ist, schaltet man das vergrössernde Objectiv ein und richtet es nach einem entfernten Object, das durch die obere Ebenenfläche der Halbkugel gespiegelt wird. Ist diese zu ibrer Drehaxe normal, so wird das gespiegelte Object seine Lage nicht ändern, wenn die spiegelnde Ebenenfläche um ibre Axe gedreht wird. Wird eine kleine Verschiebung des Bildes beobachtet, so nehme man die Hălfte derselben durch die Justirschrauben ab und wiederhole die Prufung. Mit dem Abbe-Pulfrich'schen Apparate richtete ich das Fernrobr nach der Kuppel der Frauenkirche in Munchen, welche etwa $250 \mathrm{~m}$ weit von dem Beobachtungsorte liegt. Ich bemerkte im Fadenkreuze des Fernrohres eine sehr kleine Verschiebung, welche nach Augenmaass auf kaum einige Millimeter taxirt werden konnte. Wäre die Verschiebung $10 \mathrm{~mm}$ gewesen, so wttrde man einen Fehler bekommen von

$$
\frac{10}{250000}=11^{\prime \prime}
$$

welcher durchaus zu vernachlässigen ist.

Das Abbe-Pulfrich'sche Refractometer brauchte also in dieser Hinsicht nicht berichtigt zu werden.

Ad 2. Die Visirlinie des Fernrohres muss senkrecht zu ihrer Drehaxe, d. h. parallel zum Verticalkreisestehen, worauf die Ablesung des Verticalwinkels gemacht wird.

Wir nehmen an, $\varepsilon_{1}$ sei eine kleine Neigung der Visirlinie in Bezug auf den Verticalkreis, Fig. 3. Ferner sei $\varphi^{\prime}$ irgend ein abgelesener Höhenwinkel. Die Richtung der Visirlinie wird $A C$ sein und sie schliesst einen Winkel $\varphi$ mit der Normalen an der oberen Ebenenfläche der Halbkugel ein. Wir werden offenbar haben: 


$$
\cos \varphi=\cos \varphi^{\prime} \cdot \cos \varepsilon_{1},
$$

wo naturlich $\varphi>\varphi^{\prime}$ ist.

Um $\varepsilon_{1}$ zu bestimmen, verfährt man am besten auf folgende Weise :

Man bringt ein planparalleles Glas auf die obere Ebenenfläche der Halbkugel; die Glasplatte wird vorerst vertical gestellt, was sehr einfach dadurch geschieht, dass man das Fernrobr etwas unter den Horizont neigt und auf ein durch die Glasplatte gespiegeltes entferntes Object einrichtet. Ist die Platte vollkommen vertical, d. h. parallel zur Verticaldrehaxe, so darf das Bild des eingestellten Objectes auch dann keine Verschiebung erleiden, wenn die Glasplatte um $180^{\circ}$ gedreht und das Fernrohr nicht verruckt wird. Im entgegengesetzten Falle wird die Hälfte der Verschiebung durch eine kleine Neigung der Glasplatte abgenommen. Nachdem dies

Fig. 3.

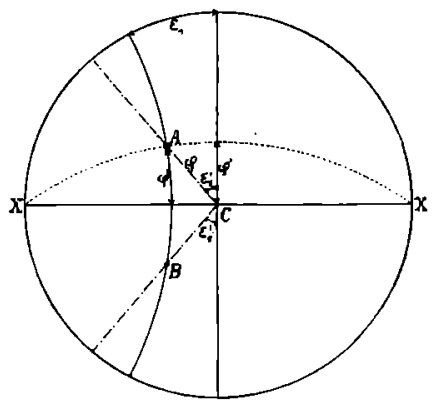
gepruft worden ist, stelle man das Fernrohr nicht gerade horizontal, aber jedenfalls so tief, dass noch ein in die Glasplatte und gleichzeitig in die obere Ebenenfläche der Halbkugel gespiegeltes entferntes Object gesehen und eingestellt werden kann. Nachber wird die Glasplatte abgenommen, das Fernrohr umgeschlagen und wieder so tief geneigt, dass das nur von der oberen Ebenenfläche der Halbkugel gespiegelte entfernte Object wieder gesehen und eingestellt werden kann.

Bewegt sich die Visirlinie wirklich in einer Meridianebene, welche durch die Verticalaxe geht, so muss nach dem Umschlagen des Fernrohres das Objectbild wieder im Kreuzpunkte genau einspielen. Bemerkt man dagegen eine kleine Verschiebung des Bildes, so wird die Hälfte derselben $\varepsilon_{1}{ }^{\prime}$ durch die Justirung weggenommen und die Prufung wiederholt. Die Justirung besteht entweder in einer kleinen Verschiebung des Fadenkreuzes, oder in der Drehung des am Objectiv angebrachten Prismas.

Das Fernrobr des Abbe-Pulfrich'schen Apparates richtele ich auf ein Dach, das von dem Beobachtungsfenster des mineralogischen Institutes etwa $100 \mathrm{~m}$ entfernt liegt. Es wurde eine kleine Verschiebung des Bildes beim Umschlagen des Fernrohres bemerkt, welche auf circa $8 \mathrm{~mm}$ geschätzt werden konnte. Daher haben wir, Fig. 3 :

$$
\varepsilon_{1}^{\prime}=\frac{\frac{8}{2}}{100000}=15^{\prime \prime} \text {. }
$$

Und da der abgelesene Winkel $\varphi^{\prime}=63^{\circ}$ betrug, so erhalten wir aus der Formel $\operatorname{tg} \varepsilon_{1}=\operatorname{tg} \varepsilon_{1}^{\prime} \cdot \sin \varphi^{\prime}$ :

$$
\operatorname{tg} \varepsilon_{1}=5,55194 ; \varepsilon_{1}=12^{\prime \prime} \text {. }
$$


Für Höhenwinkel, welche von $50^{\circ}$ bis $90^{\circ}$ gehen, bewirkt der Fehler von circa 12" keinen merklichen Einfluss, wie aus der Formel

zu schliessen ist.

$$
\cos \varphi=\cos \varphi^{\prime} \cdot \cos \varepsilon_{1}
$$

Ich habe daher auch in dieser Hinsicht keine Justirung des Apparates vorgenommen.

Ad 3. Die Drehaze des Fernrohres muss zuroberen Ebenenfläche der Halbkugel parallel sein.

Fig. 4.

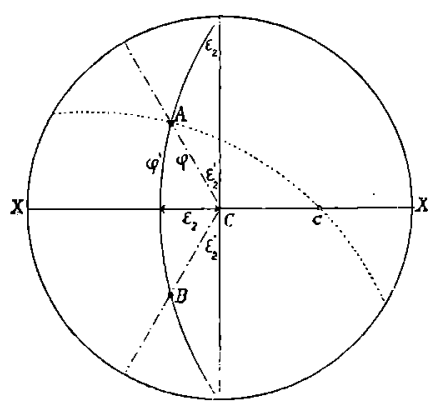

Wir benennen eine sehr kleine Neigung der Drehaxe mit $\xi_{2}$, Fig. 4 .

Wegen dieses Winkelfehlers bewegt sich die Visirlinie nicht in einer durch die Verticalaxe gehenden Meridianebene, sondern in einer solchen, welche mit der Verticalaxe den kleinen Winkel $\varepsilon_{2}$ einschliesst. Es sei $\varphi^{\prime}$ wiederum der am Verticalkreise abgelesene Winkel und $\varphi$ derjenige, welcher von der Visirlinie mit der Verticalaxe gebildet wird.

Um $\varepsilon_{2}$ zu bestimmen, wird wie vorhin verfahren. Man lege eine planparallele Glasplatte senkrecht auf die obere Ebenenfläche der Halbkugel und jichte das Fernrohr auf das gespiegelte Bild eines sehr hoch gelegenen Objectes. Ist die Einstellung gemacht, so wird die Glasplatte weggenommen, das Fernrohr umgeschlagen und auf das von der Ebenenfläche der Glaskugel erzeugte Bild desselben Objectes gerichtet. Ist ein Fehler $\varepsilon_{2}$ vorhanden, so wird es naturlich unmöglich sein, das Bild nach dem Umschlagen des Fernrobres wieder in den Kreuzpunkt desselben zu bringen. Ich habe das Fadenkreuz des Fernrohres diesmal mit der Mitte eines auf dem Dache des Akademiegebäudes befindlichen Porzellanglöckchens des Telephons in Goincidenz gebracht. Die Entfernung habe ich auf ca. $150 \mathrm{~m}$ geschätzt. Die Verschiebung des Bildes, nach Umschlagen des Fernrohres, hat ungefähr $12 \mathrm{~mm}$ betragen. Daraus :

$$
\varepsilon_{2}^{\prime}=\frac{\frac{12}{2}}{150000}=15^{\prime \prime} \text {. }
$$

Und da $\varphi^{\prime}=40^{\circ}$ war, so bekommen wir aus der Formel

den Werth

$$
\sin \varepsilon_{2}=\operatorname{tg} \varepsilon_{2}^{\prime} \cdot \operatorname{tg} \varphi^{\prime}
$$

$$
\varepsilon_{2}=12^{\prime \prime} \text {. }
$$

Auch dieser Fehler wurde naturlich vernachlässigt.

Aus der gemachten Prufung geht hervor, mit welcher Sorgfalt der Abbe-Pulfrich'sche Apparat in der Zeiss'schen Werkstatt construirt und justirt worden ist. 
Wir bemerken noch, dass, wenn beide Fehler $\varepsilon_{1}$ und $\varepsilon_{2}$ gleichzeitig auftreten, die Formeln gelten:

und

$$
\cos \varphi=\cos \varepsilon_{1} \cos \varepsilon_{2} \cos \varphi^{\prime}+\sin \varepsilon_{1} \sin \varepsilon_{2}
$$

$$
\cos \varepsilon^{\prime}=\frac{\sin \varphi^{\prime}}{\sin \varphi} \cdot \cos \varepsilon_{1}
$$

worin $\varepsilon^{\prime}$ die Winkelverschiebung des

Fig. 5.

Bildes beim Umschlagen des Fernrohres bedeutet, Fig. 5 .

Fur $\varphi^{\prime}=90^{\circ}$, d. h. wenn das Fernrohr horizontal oder nahezu horizontal gestellt ist, wird auch $\varphi$ nahezu $90^{\circ}$, und folglich $\varepsilon^{\prime}=\varepsilon_{1} ;$ d. h. die horizontale Verschiebung des Bildes wird nur von dem Fehler $\varepsilon_{2}$, nämlich von der Neigung der Visirlinie gegen den Verticalkreis, abhängen.

Wir durfen und müssen daher bei

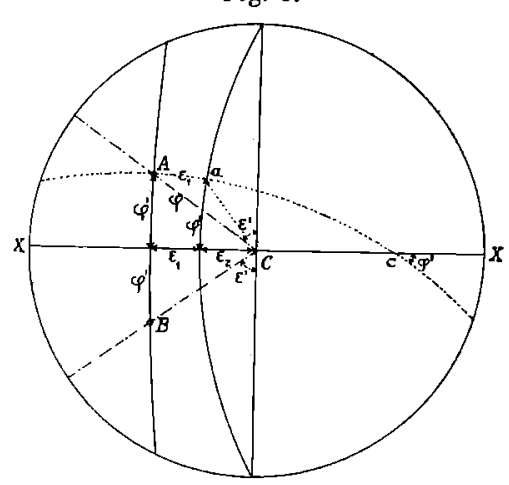
der Prufung und Correction des Apparates vorerst den Fehler $\varepsilon_{1}$ wegbringen, indem man das Fernrohr horizontal oder nahezu horizontal stellt; erst dann wird das Refractometer auf $\varepsilon_{2}$ gepruft und rectificirt, indem man Fernrohr ziemlich hoch hält.

Der Nullpunkt des Verticalkreises.

Um den Höhenwinkel direct ablesen zu können, muss der Nullpunkt des getheilten Verticalkreises bekannt sein. Wäre das nicht der Fall, so wurde man gezwungen, bei jeder Bestimmung des Brechungsindex zweimal einzustellen und abzulesen, und zwar einmal mit dem Fernrohr nach links, das zweite Mal mit demselben nach rechts.

Um diese Unannehmlichkeit zu vermeiden, habe ich den Nullpunkt des Apparates bestimmt. Er ist bekanntlich diejenige Ablesung des Verticalkreises, bei welcher die Visirlinie genau vertical nach unten steht. Das einfachste Verfahren wäre eigentlich folgendes:

Man richtet das Fernrohr vertical nach oben, und man sieht zu, dass das durch die obere Ebenenfläche der Halbkugel erzeugte Bild des Fadenkreuzes im Fadenkreuz selbst einspielt. Bei dem Abbe-Pulfrich'schen Refractometer lässt sich aber dies Verfahren nicht durchfahren, da durch eine am Ocular angebrachte Glasplatte das Fadenkreuz nicht genügend beleuchtet werden konnte.

Man könnte auch so verfabren, dass man das Fernrohr schief bält, gegen ein in der oberen Ebenenfläche der Halbkugel gespiegeltes entferntes Object richtet, den ganzen Apparat umschlägt, und das Fernrohr wieder auf dasselbe Bild einstellt. 
Da sich der Höbenwinkel nicht geändert hat, so geben die zwei Ablesungen links und rechts den Nullpunkt des Apparates an. Aber auch dies Verfahren ist nicht durchführbar, da die eine Hälfle des Verticalkreises nicht getheilt ist.

Es blieb mir nichts anderes ubrig, als einen Tropfen Monobromnaphtalin auf die Mitte der Ebenenfläche der Halbkugel zu bringen und die Grenze der Totalreflexion zu beobachten; und zwar wurde das Fernrohr bald rechts, bald links eingestellt und jedes Mal abgelesen.

Nach wiederholten Messungen hat sich herausgestellt, dass der Nullpunkt des Verticalkreises auf $+\mathbf{3 7}$ " steht; d. h. man muss die links abgelesenen Winkel um 37" vermehren, um richtige Winkel zu erbalten. Naturlich wäre es ein Leichtes gewesen, durch eine kleine Verschiebung des Fadenkreuzes den Nullpunkt auf $0^{\circ}$ zu bringen.

Das Arbeiten mit dem Abbe-Pulfrich'schen Refractometer.

Um mit dem Abbe-Pulfrich'schen Apparate oder uberhaupt mit einem nach demselben Principe construirten Refractometer richtige Resultate erhalten zu können, nachdem der Apparat selbst rectificirt worden ist, muss die Ebenenfläche des zu untersuchenden Minerals zu der Ebenenfläche der Halbkugel oder resp. zu der Ebenenfläche des verwendeten Trägers genau parallel sein.

Fig. 6.

Es sei $x$, Fig. 6, die kleine Neigung, welche offenbar

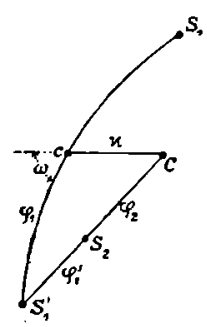
nie zu vermeiden ist. $C$ sei also der Pol der Ebenenfläche der Halbkugel und $c$ derjenige der Krystallfäche, und es mag mit $\varphi_{1}$ der Grenzwinkel der Totalreflexion zwischen der zu bestimmenden Substanz und Bromnaphtalin bezeichnet werden, deren Lichtbrechungsindices $n_{1}$ (für die bestimmte, in die anslossende Ebenenfläche beider Mittel fallende Richtung) und $n_{2}$ sind. In der Fig. 6 bedeutet: $S_{1}$ den Pol des einfallenden Strahles, $S_{1}{ }^{\prime}$ denjenigen des zutretenden Strahles. rückgeworfenen und $S_{2}$ den Pol des in die Glaskugel ein-

Die Richtung der Totalreflexion wird mit der Normalen der oberen Ebenenfläche der Halbkugel nicht denselben Winkel $\varphi_{1}$ einschliessen, sondern einen anderen Winkel, $\operatorname{der} \varphi_{1}^{\prime}$ sein mag und von $\varphi$ sehr wenig verschieden ist.

Von diesem Winkel können wir Folgendes schreiben: $\cos \varphi_{1}{ }^{\prime}=\cos \varphi_{1} \cos \varkappa-\sin \varphi_{1} \sin \varkappa \cos \omega$, wenn $\omega$ das Azimuth bedeutet.

$$
\begin{aligned}
\text { Fur } \omega=0^{0} \text { ist } \varphi_{1}^{\prime}=\varphi_{1}+\varkappa \\
-\omega=180^{\circ}-\varphi_{1}^{\prime}=\varphi_{1}-x
\end{aligned}
$$

Der Winkel $\varphi_{1}^{\prime}$ liegl also stets zwischen $\varphi_{1}+\varkappa$ und $\varphi_{1}-\chi$. 
Fur Werthe von $\varphi_{1}$, welche nicht sehr klein sind, und da $x$ selbst sehr klein ist, ist $\varphi_{1}{ }^{\prime}=\varphi_{1}$ fur ungefähr $\omega=90^{\circ}$.

Wichtig ist noch zu bemerken, dass, wenn fur irgend welchen Werth von $\omega$

wird, auch

$$
\varphi_{1}^{\prime}=\varphi_{1}+x^{\prime}
$$

$$
\varphi_{1}^{\prime}=\varphi_{1}-x^{\prime} \text { für } \omega+180^{\circ} \text { sein muss. }
$$

Wir setzen also im Allgemeinen

wo

$$
\varphi_{1}^{\prime}=\varphi_{1} \pm x^{\prime}, \quad \text { ist. }
$$

Die Richtung der Totalreflexion tritt in die Glaskugel unter einem Refractionswinkel $\varphi_{2}$, welchen wir direct ablesen. Ist $n_{3}$ das Brechungsvermögen der Glaskugel, so erhalten wir die Beziehung

woraus hervorgeht

$$
\sin \varphi_{2}=\sin \left(\varphi_{1} \pm x^{\prime}\right) \cdot \frac{n_{2}}{n_{3}}
$$

da $x$ sehr klein ist.

$$
\sin \varphi_{2}=\frac{n_{1}}{n_{3}} \pm \cos \varphi \cdot x^{\prime} \cdot \frac{n_{2}}{n_{3}}
$$

Beobachten wir also die Grenzlinie der Totalreflexion in einer bestimmten Stellung der Glaskugel und bezeichnen wir den abgelesenen Winkel mit $\varphi_{2}^{\prime}$. Drehen wir die Glaskugel um 1800, stellen wir das Fernrohr wieder auf die Grenzlinie ein und bezeichnen den abgelesenen Winkel mit $\varphi_{2}^{\prime \prime}$. Wir können folgende zwei Beziehungen niederschreiben :

$$
\begin{aligned}
& \sin \varphi_{2}^{\prime}=\frac{n_{1}}{n_{3}}+\cos \varphi \cdot x^{\prime} \cdot \frac{n_{2}}{n_{3}} \\
& \sin \varphi_{2}^{\prime \prime}=\frac{n_{1}}{n_{3}}-\cos \varphi \cdot x^{\prime} \cdot \frac{n_{2}}{n_{3}},
\end{aligned}
$$

deren Summe Folgendes giebt:

$$
\frac{\sin \varphi_{2}{ }^{\prime}+\sin \varphi_{2}^{\prime \prime}}{2}=\frac{n_{1}}{n_{3}} .
$$

Aus dem Winkelwerthe von zwei Ablesungen können wir also $n_{1}$ berechnen. Es lässt sich das Verfahren vereinfachen, wenn wir bedenken, dass $x$ sehr klein ist, und daher $\varphi_{2}^{\prime}$ und $\varphi_{2}{ }^{\prime \prime}$ sebr wenig von einander verschieden sind.

Gehen wir von der Beziehung :

aus, wo wir

$$
\sin (A+B)+\sin (A-B)=2 \sin A \cdot \cos B
$$

$$
A+B=\varphi_{2}^{\prime} \quad \text { und } \quad A-B=\varphi_{2}^{\prime \prime}
$$

setzen. Es ist daher

$$
A=\frac{\varphi_{2}^{\prime}+\varphi_{2}^{\prime \prime}}{2} \text { und } B=\frac{\varphi_{2}^{\prime}-\varphi_{2}^{\prime \prime}}{2}
$$

Groth, Zeitschrift f. Krystallogr. $X X X$. 
und da $B$ sehr klein ist, so ist sein Cosinus $=1$. Folglich :

$$
\frac{\sin \varphi_{2}^{\prime}+\sin \varphi_{2}^{\prime \prime}}{2}=\sin \left(\frac{\varphi_{2}^{\prime}+\varphi_{2}^{\prime \prime}}{2}\right)
$$

und

$$
\sin \left(\frac{\varphi_{2}^{\prime}+\varphi_{2}^{\prime \prime}}{2}\right)=\frac{n_{1}}{n_{3}} \text {. }
$$

Wir fassen folgender Weise zusammen:

Um das Lichtbrechungsvermögen zu bestimmen, werden zwei Einstellungen gemacht, indem die Glaskugel um $180^{\circ}$ gedreht wird. Das arithmetische Mittel der zwei Ablesungen ist der wahre Winkel der Totalreflexion, und nach der Formel

$$
\sin \left(\frac{\varphi_{2}^{\prime}+\varphi_{2}^{\prime \prime}}{2}\right)=\frac{n_{1}}{n_{3}}
$$

wird $n_{1}$ berechnet, naehdem $n_{3}$ bekannt ist.

Eine Stelle tritt offenbar ein, wo $\varphi_{2}^{\prime}=\varphi_{2}^{\prime \prime}$ ist. Darauf senkrecht erlangt die Differenz $\varphi_{2}^{\prime}-\varphi_{2}^{\prime \prime}$ ibren maximalen Werth.

Fur die Kenntniss des Lichtbrechungsvermögens eines zweiaxigen Krystalles sind drei Hauptindices, $\alpha, \beta, \gamma$, zu bestimmen. Dafur ist bekanntlich eine einzige Fläche des Krystalles genugend ${ }^{1}$ ).

Fur jede Fläche treten stets zwei Grenzlinien auf. Jede derselben hat ein Maximum und ein Minimum. Das grösste Maximum ist offenbar $\gamma$, das $k$ leinste Minimum ist offenbar $\alpha$. Einer der zwei anderen Werthe, Maximum oder Minimum, ist $\beta$. Die Zweideutigkeit verschwindet, wenn man angenähert die Richtung der Fläche kennt, oder wenn man uber einen anderen Schnitt des Krystalles verfugt.

Für den Albit von Lakous habe ich verschiedene Schnitte parallel zu (010) und (001) gebabt, uberdies noch einen Schnitt in der Zone [100], den mir Voigt \& Hochgesang in Göttingen verfertigten.

In den Schnitten parallel zu (010) sind Zwillingsstreifungen nicht vorgekommen; man konnte sich davon leicht uberzeugen, da der Schnitt nur zwei Grenzlinien zeigte. Sie konnten mit und ohne Nicol scharf gesehen und eingestellt werden. Die anderen Schnitte zeigten mehrere Grenzlinien, bald drei, bald vier; sie enthalten ein Gemisch von Zwillingen. Das war aber durchaus kein Hinderniss für die Untersuchung; im Gegentheil konnte man durch einen Schnitt allein, ohne seine Lage zu kennen, alle drei Werthe $\alpha, \beta, \gamma$ bestimmen, und zwar nicht einmal, sondern zwei- oder dreimal.

Wenn mit dem Abbe-Pulfrich' schen Refractometer die Werthe $\alpha, \beta$, $\gamma$ eines Krystalles mit grosser Genauigkeit bestimmt werden können, können ihre Differenzen, d. h. die Doppelbrechung, mit noch grösserer Schärfe ge-

1) Man vergleiche auch in: C. Pulfich as Totalreflectometer und das Refractometer für Chemiker etc. Leipzig 1890. 
messen werden. Denn für die Bestimmung der Differenzen ist eine Mikrometer'schraube angebracht ${ }^{1}$ ), welche in 200 Theile eingetheilt ist, wo ein Theilstrich 6" entspricht. Dieselbe Methode eignet sich ausgezeichnet für die Dispersionsbestimmung. Sind $N$ Theile auf der Mikrometertrommel abgelesen worden, so wird der entsprechende Winkel $\Delta$ berechnet nach der Formel : $\operatorname{tang} A=N \cdot \operatorname{tang} 6^{\prime \prime}$.

\section{Brechungsvermögen und Dispersion des $A b b b^{\prime}$ schen Glases.}

Für die Bestimmung der Brechungsindices der Halbkugel wandte ich einen wasserhellen Kalkspath an, dessen Brechungsvermögen sehr genau, ganz besonders durch die neue Arbeit von Sarasin, bekannt ist.

Da aber der Lichtbrechungsindex des Monobromnaphtalins für $\mathrm{Na}$ $=1,6582$ ist, während derjenige für den ordinăren Strabl des Galcits grösser ist, so konnte ich nur von dem extraordinären Strahle des. Galcits Gebrauch machen. Bei der Einstellung war das Minimum immer $=\varepsilon$.

Ich bestimmte vorher durch zablreiche Messungen das Lichtbrechungsvermögen fur $\mathrm{Na}$ oder fúr die $D$-Linie.

Es ergab sich als Mittel der Grenzwinkel

$$
51051^{\prime} 12^{\prime \prime} \text {. }
$$

Fur Kalkspath angenommen

ergab sich fur die Glaskugel

$$
\varepsilon=1,48644
$$

$$
n_{D}=1,89010 \text {. }
$$

Wurde nachher mit dem Sonnenspectrum gearbeitet, so wurde das Fernrohr auf $52^{\circ}$ festgeklemmt und alle Einstellungen mit Hülfe der Mikrometerschraube abgelesen resp. berechnet.

Schreiben wir hier die Indices für den Kalkspath, wie ich sie angenommen hatte :

$\begin{array}{lr}A & \varepsilon=1,48260 \\ a & 1,48336 \\ B & 1,48391 \\ C & 1,48474 \\ D & 1,48644 \\ E & 1,48868 \\ b & 1,48903 \\ F & 1,49080 \\ G & 1,49470\end{array}$

so erhielt ich fur die Abbe'sche Glaskugel folgende Werthe :

1) Man sebe auch: C. Pulfich, Veber Dispersionsbestimmung nach der Totalreflexionsmethode mittelst mikrometrischer Messung. Zeilschr. f. Instrumentenk. 1893, Heft 7. 


$\begin{array}{lr}A & n=1,86550 \\ a & 1,87080 \\ B & 1,87450 \\ C & 1,87896 \\ D & 1,89010 \\ E & 1,90500 \\ b & 1,90800 \\ F & 1,91892 \\ G & 1,94250\end{array}$

Ich habe diese Zahlen, sowie diejenigen, welche sich auf Calcit beziehen, in Tafel VIII aufgetragen. Die Abscissen geben die Lage der Fraunhoferc hen Linien an, auf die Ordinaten sind die Zahlen $n$ aufgetragen, und die so erhaltenen Punkte sind durch eine continuirliche Curve verbunden worden. Diejenige fur die Glaskugel ist mit einem halb so grossen Maassstabe als diejenige für den Kalkspath gezeichnet.

Brechungsindices und Dispersion des Albits.

Dasselbe Verfahren ist auch fur Albit angewandt worden. Vorher ergaben sich als mittlere Werthe für $\mathrm{Na}$-Licht oder die $\mathrm{D}$-Linie

$$
\begin{array}{llll}
54^{0} & 29^{\prime} & 26^{\prime \prime} & \text { Max. } \\
54 & 12 & 57 & \text { Mittel } \\
53 & 59 & 46 & \text { Min. }
\end{array}
$$

Daraus berechnet sich

$$
\begin{gathered}
\alpha_{D}=1,52903 \\
\beta_{D}=1,53330 \\
\gamma_{D}=1,53858 \\
\gamma-\beta=0,00528, \beta-\alpha=0,00427, \gamma-\alpha=0,00955 .
\end{gathered}
$$

\begin{tabular}{|c|c|c|c|}
\hline & $\alpha$ & $\beta$ & \\
\hline$A$ & $54^{0} 44^{\prime} 51^{\prime \prime}$ & $54053^{\prime} 43^{\prime \prime}$ & $55^{0} \quad 9^{\prime} \quad 5^{\prime \prime}$ \\
\hline 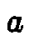 & $\begin{array}{lll}54 & 33 & 22\end{array}$ & $54 \quad 4534$ & 55126 \\
\hline$B$ & $54 \quad 2649$ & $54 \quad 40 \quad 25$ & $\begin{array}{lll}54 & 5723\end{array}$ \\
\hline$C$ & $54 \quad 19 \quad 54$ & $\begin{array}{llll}54 & 32 & 48\end{array}$ & $\begin{array}{llll}54 & 50 & 24\end{array}$ \\
\hline$D$ & $\begin{array}{lll}53 & 59 & 46\end{array}$ & 541257 & $54 \quad 2926$ \\
\hline$E$ & $\begin{array}{lll}53 & 32 & 22\end{array}$ & $\begin{array}{lll}53 & 45 & 23\end{array}$ & $54 \quad 118$ \\
\hline$b$ & $53 \quad 26 \quad 55$ & $\begin{array}{lll}53 & 39 & 52\end{array}$ & $5355 \quad 50$ \\
\hline$F$ & $\begin{array}{lll}\mathbf{5 3} & \mathbf{5} & \mathbf{2 7}\end{array}$ & $\begin{array}{llll}53 & 19 & 34\end{array}$ & $\begin{array}{lll}53 & 36 & 36\end{array}$ \\
\hline & $\begin{array}{lll}52 & 30 & 37\end{array}$ & $\begin{array}{llll}52 & 43 & 28\end{array}$ & $\begin{array}{lll}52 & 58 & 20\end{array}$ \\
\hline
\end{tabular}

Diese Werthe als Grundlage angenommen, wurden die übrigen Einstellungen mit Hülfe der Mikrometerschraube bestimmt.

Ich erbielt folgende Zusammenstellung:

aus welchen Winkeln folgende definitive Werthe fur Albit von Lakous sich ergeben: 
Ueber einige im mineral. Instilute zu Minchen ausgeführte Untersuchungen. 437

$\begin{array}{lrrr}A & \alpha=1,52340 & \beta=1,52618 & \gamma=1,53095 \\ a & 1,52411 & 1,52795 & 1,53292 \\ B & 1,52505 & 1,52935 & 1,53468 \\ C & 1,52648 & 1,53058 & 1,53614 \\ D & 1,52905 & 1,53330 & 1,53858 \\ E & 1,53213 & 1,53640 & 1,54160 \\ b & 1,53274 & 1,53701 & 1,54224 \\ F & 1,53550 & 1,53987 & 1,54553 \\ G & 1,54130 & 1,54571 & 1,55095\end{array}$

Zuverlässige Werthe sind die, welche sich auf die Fraunhofer'schen Linien $B, C, D, E, b$ und $F$ beziehen. Die Linien $A$, $a$ und $G$ konnten nicht scharf erhalten werden, und daher war ihre Einstellung mulhsam, schwer und nicht vollkommen sicher.

Der Gang der continuirlichen Curven für Albit, welche in Tafel VIII gezogen sind, lässt aber auf die wahrscheinlichsten Werthe des Brechungsindex fur die Fraun hofer'schen Linien $A, a$ und $G$ schliessen.

Da die Bestimmung der Doppelbrechung des Lichtes, wie sie aus der Abbe'schen Methode hervorgeht, eine sehr genaue genannt werden kann, so ist es erlaubt, den Winkel der optischen Axen aus den Werthen der Brechungsindices direct zu berechnen.

\begin{tabular}{|c|c|}
\hline$A$ & $2 V=74^{\circ} 56^{\prime}$ \\
\hline$a$ & 8252 \\
\hline$B$ & 8144 \\
\hline$C$ & 8134 \\
\hline$D$ & $84 \quad 4$ \\
\hline$E$ & 8438 \\
\hline$b$ & 8428 \\
\hline$F$ & 8254 \\
\hline$G$ & 8520 \\
\hline
\end{tabular}

Und es ergiebt sich sehr deutlich

$$
\varrho<v \text { (bezogen auf } \mathfrak{c} \text { ). }
$$

\section{Genauigkeit der Resultate.}

Um die Genauigkeit der Ergebnisse beurtheilen zu können, müssen wir die einzelnen Beobachtungswerthe zusammenstellen, welche die Brechungsindices für die $D$-Linie gegeben haben. Dabei wurden folgende Schnitte des Albits verwendet:

drei parallel der Spaltung (001), vier - - - $(010)$ und

zwoi Schnitte in der Zone [001].

Es folgt die Zusammenstellung der Winkel und der daraus sich ergebenden mittleren Fehler 


\begin{tabular}{|c|c|c|c|c|c|}
\hline für & $\alpha$ & & $\beta$ & & $\gamma$ \\
\hline & 54029 & $0^{\prime \prime}$ & 一 & & - \\
\hline & $54 \quad 29$ & 15 & - & & - \\
\hline & 5428 & 2 & - & & - \\
\hline & 5429 & 0 & $54011^{\prime} 54^{\prime \prime}$ & 540 & $\begin{array}{lll}0 & 0^{\prime} & 0^{\prime \prime}\end{array}$ \\
\hline & 5430 & 10 & $54 \quad 1130$ & 535 & 5630 \\
\hline & $54 \quad 30$ & 0 & $54 \quad 1226$ & 535 & 5738 \\
\hline & $54 \quad 27$ & 30 & $54 \quad 12 \quad 46$ & 535 & 5936 \\
\hline & 5427 & 54 & $54 \quad 11 \quad 39$ & 535 & $58 \quad 42$ \\
\hline & $54 \quad 28$ & 325 & $54 \quad 12 \quad 54$ & 53 & 5946 \\
\hline & $54 \quad 29$ & $\begin{array}{l}9 \\
0\end{array}$ & $54 \quad 12 \quad 58$ & 54 & 127 \\
\hline & $\begin{array}{r}54028 \\
\pm 5\end{array}$ & & $\begin{array}{c}54^{0} 12^{\prime} 17^{\prime \prime} \\
\pm 37^{\prime \prime}\end{array}$ & $\begin{array}{c}53^{0} \\
\pm\end{array}$ & $\begin{array}{l}59^{\prime} 7^{\prime \prime} \\
1^{\prime} 38^{\prime \prime}\end{array}$ \\
\hline
\end{tabular}

Aus diesen Zahlen lassen sich die mittleren Febler der Indices berechnen, nämlich :

$$
\begin{aligned}
& \alpha=1,52905 \pm 0,00050 \\
& \beta=1,53330 \pm 0,00020 \\
& \gamma=1,53858 \pm 0,00028^{1} \text { ) }
\end{aligned}
$$

Aus dem Gesagten geht deutlich bervor, dass das mit Hulfe des AbbePulfrich'schen Refractometers bestimmte Lichtbrechungsvermögen eine unvergleichlich kleinere Genauigkeit besitzt, als die mit demselben Apparate bestimmte Doppelbrechung, da diese letztere sogar bis auf die fünfte Decimalstelle angegeben werden kann, was durch Messung der Differenzwinkel mit Hülfe einer am Apparate angebrachten Mikromelerschraube geschieht. - Ich bin aber der Meinung, dass der Abbe-Pulfrich'sche Apparat auch dazu geeignet ist, das Lichtbrechungsvermögen mit derselben Genauigkeit zu bestimmen wie die Doppelbrechung. Die unsichere Ablesung am Verticalkreise, die ungleiche Homogenilät und Krummung der Halbkugel etc. können dahin wirken, den angegebenen Fehler bei dem Brechungsvermögen hervorzurufen. Um solche beeinflussende Factoren so weit als möglich wegzubringen, genúgt es, dieselbe Methode bei der Bestimmung des Brechungsvermögens anzuwenden, welche bei der Bestimmung der Doppelbrechung schon in Anwendung ist: ich will eine solche Methode Differentialmethode nennen. Man bestimme vorher durch die Methode der kleinsten Ablenkung das Brechungsvermögen verschiedener Glassorten. - Soll das Brechungsvermögen eines Minerals vollkommen genau bestimmt werden, so wähle man von den zur Verfugung stehenden Glassorten diejenige Platte als Vergleichsobject, deren Brechungsvermögen nahe demjenigen des zu bestimmenden Minerals liegt. Handelt es sich z. B. um die Bestimmung des Brechungsvermögens von Albit, so wähle man eine Glas-

1) Dïe wahrscheinlichsten Fehler sind aber: $\pm 0,00033$ für $\alpha, \pm 0,00013$ für $\beta$ und $\pm 0,00017$ für $\gamma$. 
platte aus, deren Brechungsindex z. B. 1,52157 ist. Eine solche Glasplatte wird auf die Abbe'sche Halbkugel gelegt, das Fernrohr auf die durch die Glasplatte erzeugte Grenzlinie genau eingestellt und festgeklemmt. Alsdann wird die Glasplatte abgenommen, ohne am Apparate etwas zu ändern, und an ibrer Stelle der Albit gebracht. Da das Fernrobr nicht verschoben wurde, so wird es jetzt auf die dureh den Albit erzeugten Grenzlinien mit Hulfe der Mikrometerschraube scharf eingestellt. Die Winkeldifferenz (zwischen Glas und Albit) wird dadurch mit grosser Genauigkeit abgelesen resp. berechnet, und damit werden auch die entsprechenden Grenzwinkel des Albits bekannt, und zwar mit derselben Genauigkeit wie für die Glasplatte.

Diese Differentialmethode macht den Verticalkreis unnöthig, wodurch sich das Abbe-Pulfrich'sche Refractometer einfacher und billiger gestallet. Um ohne Verticalkreis einen Theilstrich der Trommel der Mikrometerschraube bestimmen zu könned, werden zwei verschiedene Glasplatten angewendet, deren Brechungsindex $n_{1}$ und $n_{2}$ bekannt sind. Bezeichnet man mit $N$ die Anzabl der auf der Trommel abgelesenen Theile, welche der Differenz $n_{1}-n_{2}$ entsprechen, ferner mit $x$ den unbekannten Werth eines Theilstriches der Trommel und mit $\varphi_{1}$ und $\varphi_{2}$ die berechneten Grenzwinkel der Glasplatten, dann folgt $x$ aus der Gleichung:

$$
\operatorname{tg}\left(\varphi_{1}-\varphi_{2}\right)=N \cdot \operatorname{tg} x
$$

\section{Die optische Bestimmung nach Fedorow.}

Der Fedorow'sche Tisch wird nach und nach sowohl in der Mineralogie als auch ganz besonders in der Petrographie vielfach benutzt werden, sobald das Arbeiten mit ibm sich eingebürgert haben wird, denn um mit dem Tischchen bequem arbeiten zu können, braucht man lange und mühsame Uebung.

Durch die Fedorow'sche Methode gelingt es mit gentigender Genauigkeit, die Lage der optischen Axen mit Bezug auf die krystallographischen Zonen von sehr kleinen Dünnschliffen eines Minerals festzustellen, während durch keinen anderen bekannten Apparat irgend welches Resultat in dieser Hinsicht zu erzielen ist; denn bei dem Fedorow'schen Mikroskope arbeitet man stets mit parallelem Lichte, was eben den Vorzug dieser Methode ausmacht. Will man die optische Axe im Fadenkreuz des Oculars baben, so neigt man den Schliff so weit, bis er immer dunkel bleibt, wie auch die Nicols gedrebt werden, oder besser so: steht eine optische Axe zur Axe des Mikroskopes parallel, so hat man im Gesichtsfelde bei gekreuzten Nicols nicht vollkommene Auslöschung, sondern etwas hell und zwar immer gleich bell, auch wenn die Nicols gedrebt werden, in Folge der inneren conischen Refraction. Bringt man den Schliff nur etwas aus dieser Stelle heraus, so erscheint er sofort dunkel. Die Einstellung kann in Folge dessen nicht anders als scharf sein. 
Ich werde die Methode für die optische Untersuchung der Feldspäthe hier kurz zusammenfassen. Der neue, von R. Fues s construirte Fed o row -

Fig. 7.

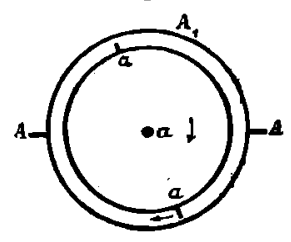
sche Tisch besteht aus vier Drehaxen, nämlich zwei inneren $a a$ und $a_{1}$ und zwei äusseren $A A$ und $A_{1}$, Fig. 7, welche auch zueinander parallel gestellt werden können. Die Axe $A A$ ist horizontal und unbeweglich, $A_{1}$ ist auf dieser senkrecht und mit ihr um $360^{\theta}$ drehbar. Die zwei inneren Axen $a a$ und $a_{1}$ haben dieselbe Aufgabe zu erfullen wie $A$ und $A_{1}$, nur mit dem Unterschiede, dass die Axe aa auch beweglich ist. Auf dem Tischchen sitzen zwei Glashalbkügelchen, das eine unterhalb, und das andere oberhalb, dieses letztere direct auf dem Präparate festgeklebt. Da das Lichtbrechungsvermögen der Halbkugelchen nahezu gleich demjenigen der Feldspäthe ist, so wird durch Neigung des Tisches um $90^{\circ}$ links und rechts fast das ganze Mineral optisch untersucht. Wäre die Brechung der Glaskugelchen etwas grösser, so würde man dieses Ziel vollkommen erreichen durch Neigung des Präparates um weniger als $90^{\circ}$.

Die Brechung des oberen Glaskugelchens habe ich mit Hulfe des Abbe'schen Refractometers bestimmt und folgende Zahl erhalten:

$$
n=1,5217 \text { fur } N a \text {. }
$$

Um die Kügelchen zu centriren, verfährt man auf folgende Weise: Man heftet vorerst das eine durch einen Tropfen Oel auf die untere Fläche des Tisches, so dass das Centrum der Halbkugel mit dem Kreuzpunkte des Tisches nabezu zusammenfallt ${ }^{1}$ ). Das andere Halbkügelchen wird auf das Präparat ebenfalls durch einen Tropfen Oel festgelegt, indem man die richtige Stelle des zu untersuchenden Präparates vorher geseben und fixirt hat. Durch eine sebr kleine Verschiebung wird der zu untersuchende Gegenstand in den Kreuzpunkt des Mikroskopes gefuhrt, und damit ist die Centrirung erreicht.

Es ist wichtig, dass die Drehaxe $A A$ durch das Präparat selbst geht, damit bei der Drehbewegung des Tisches um $A A$ es nicht gleich aus dem Gesichtsfelde heraustritt. Gelingt dies nicht durch eine Stellung des Präparates, so kippt man dasselbe um; der Fuess'sche Tisch ist so eingerichtet, dass das Deckglas nach unten stehen muss.

Ist einmal alles richtig eingestellt, so kann die Arbeit beginnen, und zwar zerfällt sie in folgende zwei Aufgaben :

1) Den Winkel der optischen Axen zu bestimmen, wenn das Mineral nicht orientirt werden kann, oder resp. die Lage der optischen Axen zu bestimmen, wenn das Mineral nicht genau orientirt werden kann;

1) Es wäre zu wünschen, dass auch die Glashalbkügelchen einen Kreuzpunkt besässen. 
2) die Lage der optischen Axen zu beslimmen, wenn der Dünnschliff orientirt ist.

Da ich nur gut orientirte Dünnschliffe von Albit hatte, die mit den Spaltungen (001) und (010) parallel waren, so werde ich mich nur mit der zweiten Aufgabe befassen. Die erste ist fur sich verständlich.

Uebrigens ist bei fast allen Dunnschliffen von Feldspäthen eine theilweise Orientirung möglich, da die Spaltung (010) oder (001) zur Axe des Mikroskopes parallel gebracht werden kann.

Ist ein Schnitt parallel (010) vorhanden, so stellt Fedorow diese Fläche nach oben und die Spaltung (001) senkrecht zur Axe $A A$. So werden diese Flächen auf das stereographiscbe Netz aufgetragen und geben den Nullpunkt beider Theilkreise des Tisches.

Um die Orientirung der Flächen, wie sie ublich ist, zu bewerkstelligen, giebt Fedorow dem stereographischen Netze die nöthigen Drehungen.

Ich babe aber gefunden, dass es am besten sei, diese Drehungen sofort dem Präparate zu ertheilen, d. h. den Flächen (001) und (010) im Mikroskope selbst diejenige Lage zu geben, wie sie bei einem orientirten Feldspathe liegen, und dazu eignen sich die zwei inneren Drehaxen $a a$ und $a_{1}$.

Ich verfabre daher so :

Im Anfange steht (010) nach oben und alle Kreise des Tisches zeigen Null. Das Präparat wird um die Axe $a_{1}$ so weit gedreht, bis die Spur (001) zur Axe $a a$ parallel steht, und diese letztere wird dann um die Axe $A_{1}$ so weit gedreht, bis sie zur Axe $A A$ senkrecht steht. Dann wird das Präparat um die Axe $a a$ um $90^{\circ}$ umgeschlagen, damit die Fläche (010) zu $A A$ senkrecht zu stehen kommt. Jetzt wird diese Axe benutzt und das Präparat um $26^{\circ}$ gedreht. Die Zählung beginnt von dieser Nullstellung des Tisches.

Hat man dagegen einen Dunnschliff parallel zur Spaltung (001), so wird die Spur (010) zu $a$ a parallel und diese Axe wie vorhin zu $A A$ senkrecht gerichtet. Die Drehung um die Axe $a a$ beträgt jetzt nur $4^{0}$ nach links oder nach rechts und die Drehung um die Axe $A A$, wie fruher, $26^{\circ}$ nach hinten oder nach vorne. Damit erreicht man dieselbe Orientirung des Feldspathes, liege der Dunnschliff (001) oder (010) auf dem Fedorow'schen Tische vor.

Nachdem die Nullstellung bekannt geworden ist, dreht man das Präparat wieder zurück, indem man nur die zwei ảusseren Drehaxen $A A$ und $A_{1}$ bewegt, und sucht die Lage einer optischen Axe, was durch Probiren rasch und bequem geschieht, indem man mit einer Hand beide Axen $A A$ und $A_{1}$ gleichzeitig und mit der anderen die Nicols hin und her dreht. Man spure immer nach derjenigen Bichtung hin, nach welcher die Doppelbrechung abnimmt. Ist die richtige Lage gefunden, so wird am Horizontalkreise der Winkel $\omega$ und am Verticalkreise der Winkel $\varphi^{\prime}$ abgelesen. Da die Brechungsindices für Albit $\beta=1,5333$ und fur das Glaskugelchen $n=$ 1,5217 ist, so berechnet man den richtigen Winkel $\varphi$ nach der Formel 
442 C. Viola. Ueb. einige im min. Inst. zu München ausgeführte Untersuchungen.

$$
\sin \varphi=\sin \varphi^{\prime} \frac{n}{\beta} .
$$

$26^{\circ}$ werden zu $\varphi$ addirt und die so erhaltene Zabl mit dem Winkel $\omega$ auf das stereographische Netz aufgetragen. Man erhält dadurch sofort die richtige Lage der optischen Axe.

Ist diese gefunden, so dreht man das Präparat um die Axe $A A$ zuruck, wobei die gefundene optische Axe in der zur Axe $A A$ senkrechten Ebene bleibt. Man bringt auch einen Faden des Oculars in diese Ebene, und durch Drehen der Nicols sucht man die Auslöschungsschiefe $\varepsilon$. Nach der bekannten Fresnel'schen Regel wird die zweite optische Axe in einer solchen verticalen Ebene liegen, welche mit der vorherbestimmten den Winkel $2 \varepsilon$ einschliesst. Wird also diese zweite Ebene senkrecht zu $A A$ gebracht durch Dreben um die Axe $A_{1}$, so braucht man nur das Präparat nach vorne oder nach hinten um die Axe $A A$ zu neigen, um die zweite optische Axe zu treffen. Durch Probiren wird die Lage dieser auch mit Leichtigkeit festgestellt.

Ich brauche die gemessenen Winkel hier nicht wiederzugeben, welche ich für den Albit erbalten hatte. Die Pole der optischen Axen des Feldspathes sind im Netze Taf. VII eingetragen. Die einzelnen Pole sind mit $a_{1}$, $a_{2}, a_{3}, a_{4}, a_{5}$ resp. $b_{1}, b_{2}, b_{3}, b_{4}$ und $b_{5}$, die mittlere Lage der optischen Axen mit $A$ und $B$ bezeichnet. Der Winkel der optischen Axen, wie er aus dem Diagramm hervorgeht, beträgt $86^{\circ}$, ist also $2^{0}$ grösser als der berechnete (s. S. 437). Der Fehler konnte aber noch grösser sein.

Wenn wir von mathematischer Genauigkeit bei dieser Bestimmung der Lage der optischen Axen sprecheo, so soll damit gemeint sein, dass dieselbe auf ein paar Grad gemessen ist, denn jede mathematische Genauigkeit wird eben durch die Grösse des begangenen Fehlers gewogen. Aber ein paar Grad in der Bestimmung der Lage der optischen Axen und des Winkels derselben lässt nichts zu wünschen ubrig. Denn durch diese Methode können zahlreiche Schliffe eines Minerals verwendet werden, und das Mittel kann jede beliebige Genauigkeit erreichen, welche man nur braucht. Keine andere Methode, ganz besonders die, welche auf Beobachtung mit convergentem Lichte begründet ist, und fur welche die gewöhnlichen Apparate verwendet werden, kann eine solche Genauigkeit beanspruchen.

Fur solches Material, wo Individuen durcheinander verwachsen sind, oder, wie ganz besonders bei den Feldspäthen, $Z$ willinge nach verschiedenen wiederbolten Gesetzen auftreten, ist die Fedorow'sche Methode vortrefflich geeignet, und jedenfalls muss sie in der Petrographie ausgezeichnete Resultate geben. 